\title{
Total Integration: The Case of Information Systems for Olympic Games
}

\author{
Dimitrios A. Beis \\ Global Event Experts
}

\begin{abstract}
The Olympic Games, the most demanding Sports Mega Event, have evolved through the years to complexity levels unparallel in any man designed process. Information Systems play the key role both as an enabler and critical performer for an Olympic Event if not surpassing at least in equal terms with the stars of the Games, the Athletes. Just data registering of records and performance of sports and athletes is not a viable option today for the Olympic Games. The total integration of all functions that make the 15 dream days of the Olympics is the target. Olympic family services, spectator services, cultural integration, media and broadcasting services, world participation and host city performance in transportation, crowd regulation and security must be supported in a first time easy to use, reliable and sub second response performance with $100 \%$ availability of all systems. The IS implemented in support of the last three Olympiads in Sydney, Athens and Beijing will be presented, critical issues of success factors analysed and expected developments projected.
\end{abstract}

